\title{
Prospective Long-Term Assessment of Sedation-Related Adverse Events and Patient Satisfaction for Upper Endoscopy and Colonoscopy
}

\author{
Beate Kilgert $^{a, b} \quad$ Lydia Rybizki $^{c}$ Michael Grottke ${ }^{c}$ Markus F. Neurath ${ }^{a, b}$ \\ Helmut Neumann ${ }^{\mathrm{a}, \mathrm{b}}$ \\ ${ }^{a}$ Department of Medicine and ${ }^{b}$ Ludwig Demling Endoscopic Center of Excellence, University Hospital Erlangen, \\ Erlangen, and ${ }^{\mathrm{C}}$ Department of Statistics and Econometrics, University of Erlangen-Nuremberg, Nuremberg, Germany
}

\section{Key Words}

Fear of pain · Sedation-related adverse events . Upper endoscopy · Colonoscopy · Patient satisfaction

\begin{abstract}
Background: Fear of pain and sedation-related adverse events are impediments for patients to attend endoscopic screening or surveillance programs. Objective: To investigate the long-term effect of different sedation protocols in patients undergoing screening or surveillance endoscopy. Moreover, motivation of patients to decline endoscopic procedures was evaluated by focusing on the patient's satisfaction, fear and pain in relation to type of sedation used. Methods: Design: A prospective, double-blind controlled trial data collection was performed by using a standardized clinical questionnaire followed by a telephone interview 3-4 weeks after the initial endoscopic procedure. Setting: The study was conducted at the Department of Medicine I at the University Hospital of Erlangen-Nuremberg. Data collection was performed during June 2012 till April 2013. Patients: Overall, 307 patients were prospectively evaluated (44.3\% female, mean age $51 \pm 17.4$ years; mean BMI $25.5 \pm 5.7) .247$ patients (80.5\%) were outpatients, 60 inpatients (19.5\%). Interventions: Endoscopic procedures were divided into five groups: (i) procedures in the upper gastrointestinal tract, (ii) complete colonoscopies, (iii) ileocolonoscopies, (iv) incomplete colonoscopies, and (v) other procedures. Main outcome mea-
\end{abstract}

surements: Patient satisfaction, fear and pain were measured in a structured and standardized clinical interview using a 6-point numerical rating scale, where 1 was 'very satisfied/ no pain' and 6 was 'very unsatisfied/unsupportable pain'. $\boldsymbol{R e}$ sults: Different types of sedation were assessed: propofol in monosedation (6.5\%), combination of propofol + meperidine $(41.0 \%)$, combination of midazolam + meperidine (48.5\%) and other combinations (3.9\%). Patient satisfaction was significantly reduced regarding fear and pain during the endoscopic procedure $(p=0.001$ and $p=0.0001$, respectively). All patients receiving propofol monosedation indicated significantly less pain in comparison to other sedation groups $(p<0.0001)$. Moreover, sedation with midazolam + meperidine increased the fear during the procedure significantly in comparison to monosedation with propofol $(p=$ 0.082). Propofol/meperidine in combination and midazol$\mathrm{am} /$ meperidine increased the probability for cardiovascular events in comparison to monosedation with propofol $(p=$ $0.005 ; p=0.039$ ). Finally, we observed significantly lower doses of propofol when used in monosedation than propofol in combination with meperidine $(p=0.066)$. Limitation: Single-center study at a tertiary referral center. Conclusions: Propofol in monosedation should preferably be used for patient sedation in screening and surveillance endoscopies. Whether this approach could also improve participation rates in screening and surveillance endoscopies requires further investigations.

(c) 2014 S. Karger AG, Basel

\section{KARGER}

E-Mail karger@karger.com www.karger.com/dig (c) 2014 S. Karger AG, Basel

0012-2823/14/0901-0042\$39.50/0
Helmut Neumann, MD, $\mathrm{PhD}$

Department of Medicine I

University of Erlangen-Nuremberg

Ulmenweg 18, DE-91054 Erlangen (Germany)

E-Mail helmut.neumann@uk-erlangen.de 


\section{Introduction}

One quarter of all deaths in Western countries is caused by cancer, and since 1975 the worldwide incidence of colorectal cancer (CRC) has constantly increased. CRC is the fourth most common cancer affecting over 401,000 men and 381,000 women per year [1]. The prognosis of CRC is dependent on the disease stage. Therefore, increasing efforts have been made to diagnose the precursor lesions of CRC and colorectal adenoma at an early stage for subsequent endoscopic therapy. Accordingly, the American College of Gastroenterology introduced the CRC screening program in 2000, an initiative which has been widely adopted by most other national societies [2]. Nevertheless, one drawback is that the majority of patients still refuse to participate in endoscopic screening and surveillance programs [3]. Fear of pain and sedationrelated adverse events are impediments for patients to attend endoscopic screening or surveillance programs. As numerous studies have shown, sedation during endoscopic procedures improves patient satisfaction, reduces fear and pain and increases the patient's acceptance to repeat endoscopic procedures [4-8]. It was found that most patients decline the endoscopic examination because there was unpleasantness of the examination, inconvenience according to the preparation, lack of symptoms, and no time [9]. In addition, other studies have identified fear of the procedure and side effects as well as pain during and after the endoscopy as remarkable reasons of patients to avoid endoscopic procedures $[10,11]$.

Of note, recommendations for general sedation in patients undergoing endoscopic procedures are not available [12]. In Canada, more than $90 \%$ of patients receive sedation during endoscopy [13], as is also the case in North America (>98\%) and Australia (100\%). In contrast, sedation rates in Europe vary between 25 and $85 \%$, in Asia between 50 and $100 \%$, and Africa between 25 and 50\%, respectively [14]. According to one recent multicenter study in 2006, the most commonly used sedative agents were midazolam (47\%) and opioids (33\%), which was also the most common combination of agents (23\%), whereas propofol as a sedation agent was only used in less than $5 \%$ of procedures [12]. In contrast to this, an international survey performed in 2010 by Riphaus et al. [15] showed that $41 \%$ of colonoscopies were performed with propofol while $31 \%$ were performed with a combination of benzodiazepines and opioids.

The aim of this prospective study was to evaluate patients' motivations to decline endoscopic procedures by focusing on patient satisfaction, fear and pain in relation to the type of sedation used.

Assessment of Sedation-Related Adverse

Events and Patient Satisfaction

\section{Methods}

Study Design

The study was conducted at the Department of Medicine I, University Hospital of Erlangen-Nuremberg. Data collection was performed during June 2012 until April 2013. The study protocol was approved by the local institutional review board at the University of Erlangen-Nuremberg. The long-term effect of different sedation protocols in patients undergoing endoscopy was evaluated in a prospective, examiner-blinded, controlled trial. Special attention was given to patient satisfaction, fear and pain during and after the endoscopic procedure. Data collection was performed using a standardized clinical questionnaire in written form during and directly after the procedure. In addition, 3-4 weeks after the endoscopic procedure the patient was contacted again via telephone for repeat interview.

\section{Inclusion and Exclusion Criteria}

Inclusion criteria were: $>18$ years, sufficient linguistic and cognitive qualifications, literacy and patient's agreement to the study. Exclusion criteria were: $<18$ years, illiteracy, limited language skills, dementia or other diseases limiting cognitive qualifications, hearing loss or deficiency, absent or refused patient's agreement and emergency examinations.

\section{Preparation, Sedation and Documentation}

Before colonoscopy, patients underwent bowel preparation according to a standard protocol which was 4 liters of KleanPrep ${ }^{\circledR}$ for outpatients and 4 liters of Oralav ${ }^{\circledR}$ for inpatients. Before the endoscopic procedure, patients were kindly asked to answer a questionnaire regarding socio-demographic data (age, gender, height, weight), the reason on how to attend the procedure and about previous examinations and their experience regarding fear, pain, type of sedation and displeasing memory to the procedure.

During the endoscopic examination, the following parameters for the second part of the survey were documented by the study nurse: name of endoscopist, type of endoscopy, severity of intervention (grade 1-3), outpatient/inpatient, ASA physical status classification system, type and dose of sedative agents, type of endoscopic intervention (e.g. puncture, ligation, dilatation, polypectomy, stenting, changing stent, biopsy), vital signs before and during the endoscopic procedure (blood pressure, oxygen saturation, heart rate) and potential side effects.

After the endoscopic procedure the study nurse completed the documentation form for the vital signs in the anesthetic recovery room located in the endoscopy unit of the hospital. Before the patient was discharged home or transferred to the general ward, he/she was given the third part of the questionnaire by asking to evaluate the endoscopic examination. Focus relied on the patient's satisfaction, fear and pain and the acceptance for repeat endoscopies.

Three to 4 weeks after the endoscopic procedure the patient was contacted again via telephone and asked about the procedure, general satisfaction and satisfaction with the endoscopy, revaluation of fear and pain during the endoscopic procedure and acceptance for repeat endoscopies. An additional important part of this survey was to evaluate potential adverse events (e.g. nausea, vomiting, dizziness, fatigue and pain) after the procedure. In the questionnaire after examination and the telephone 
Fig. 1. Groups of endoscopic procedures.

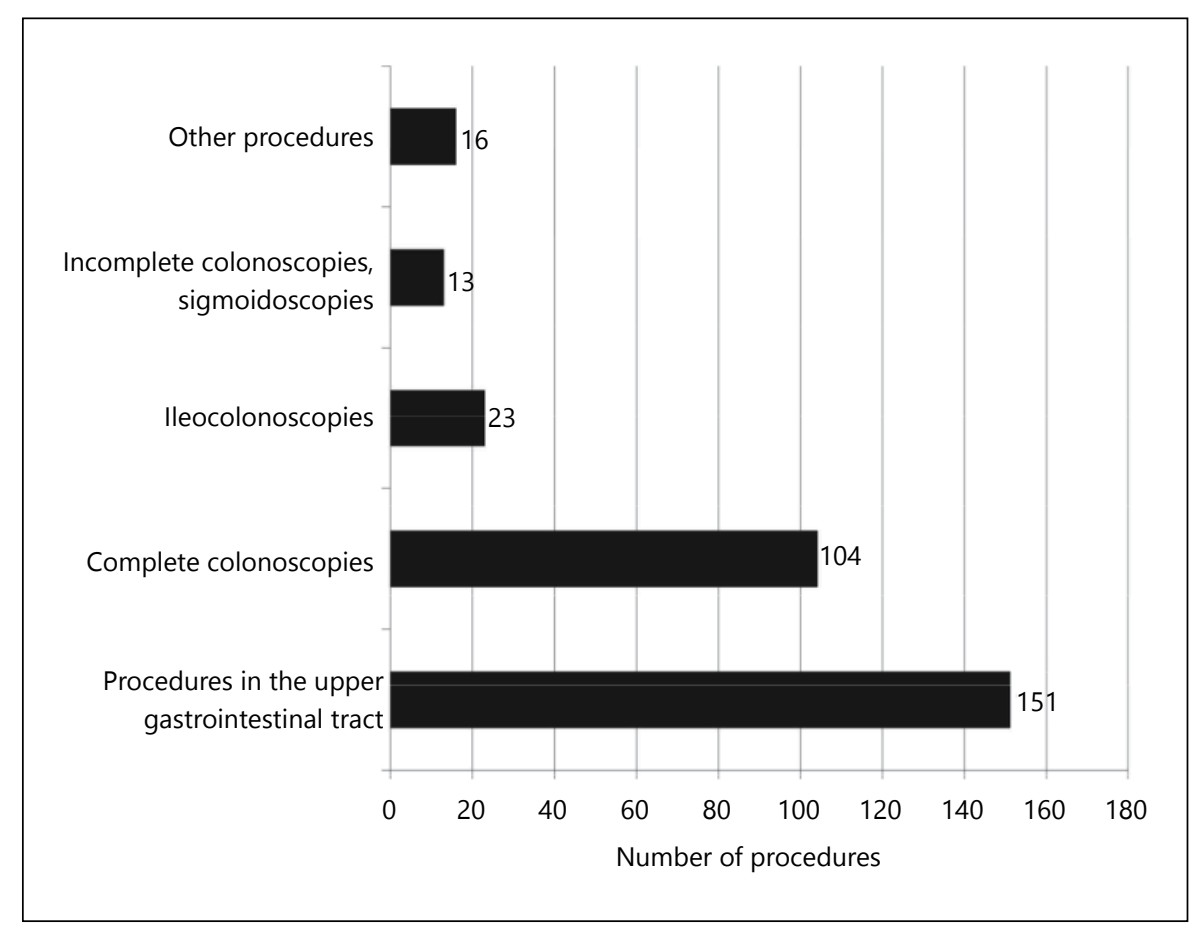

interview the patient was especially asked about problems or complaints about the procedure (administration, waiting time, other problems).

The evaluation of patient's satisfaction, fear and pain was performed by using a numerical rating scale (NRS) from 1 to 6 , where 1 was 'very satisfied/no pain' and 6 was 'very unsatisfied/unsupportable pain'. Pre- intra- and post-procedural monitoring was documented by using Siemens SC 6002XL, DINAMAP Chiromed and SIMS BCI Autocorr equipment. 17 different endoscopists ( $17.7 \%$ female) were registered with 9 main endoscopists performing the majority of endoscopic procedures.

\section{Statistical Analyses}

The statistical analysis was performed in cooperation with the Department of Statistics and Econometrics, University of Erlangen-Nuremberg. Linear and non-linear regression models were used. The single hypotheses were tested with t tests for statistical significance. The level of significance was set at $10 \%$, i.e. results with a $p$ value $<0.1$ were regarded as significant. The statistical analysis was done with $\mathrm{R}$ version 3.0.1 (including package MASS).

\section{Results}

\section{Study Population}

Overall, 307 patients were prospectively included (44.3\% female, mean age $51 \pm 17.4$ years; mean BMI $25.5 \pm 5.7), 247$ patients $(80.5 \%)$ were in ambulant treatment and 60 patients (19.5\%) were in hospital treatment.

\section{Indications, Procedures and Sedation}

Indications for an endoscopic procedure were as follows: (a) screening examination, (b) control by reason of patient's discomfort, (c) chronic inflammatory bowel disease, (d) control by reason of other disease that were polyps, Barrett's esophagus, Helicobacter pylori infection, esophageal varices and malignant disease, and (e) other indications (e.g. change of defecation or celiac disease).

Endoscopic procedures were divided into five groups: (i) procedures in the upper gastrointestinal tract, (ii) complete colonoscopies, (iii) ileocolonoscopies, (iv) incomplete colonoscopies, and (v) other endoscopic procedures (e.g. ERCP, EUS) (fig. 1).

Types of sedation were divided into the following groups (fig. 2): (a) propofol in monosedation (cumulative doses (CD) 100-440 mg, median 180, mean $199 \pm 95.5$ ); (b) combination of propofol (CD 100-980 mg, median 245, mean 271.6 \pm 140.0) + meperidine (CD 50-100 mg, median 50, mean $58.3 \pm 19.4$ ); (c) combination of midazolame (CD 3-10 mg, median 5, mean $5.4 \pm 4.2)+$ meperidine (CD 50-100 mg, median 50, mean $72.6 \pm 27.2$ ), and (d) other combinations/no sedation.

Type and dosage of sedative agents was chosen by the endoscopist who was not aware of the evaluation. The waiting time was comparable in all patients, outpatients as well as inpatients had 60 min median waiting time during which they filled out the questionnaire that took about 5-10 min. 
Fig. 2. Types of sedation used.

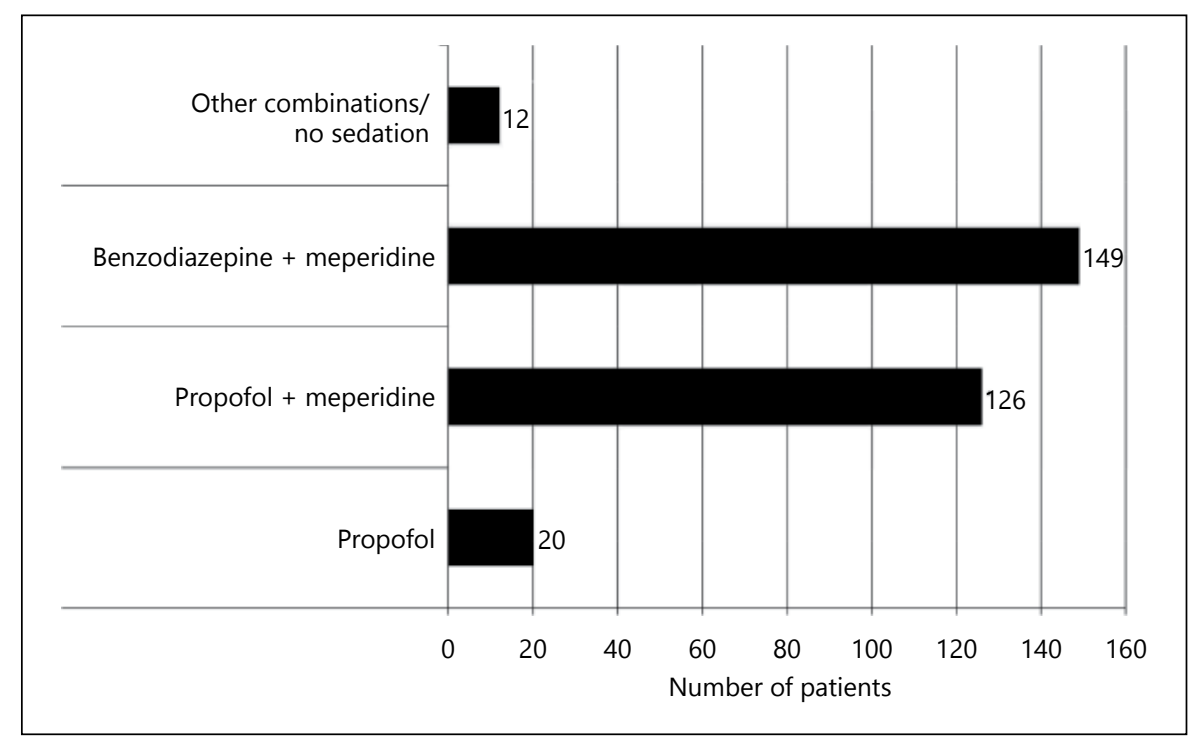

Table 1. Fear during endoscopy in different sedation groups

\begin{tabular}{|c|c|c|c|}
\hline \multirow[t]{2}{*}{ Sedation group } & \multicolumn{2}{|c|}{$\begin{array}{l}\text { Patients having fear } \\
\text { during endoscopy } \\
(\mathrm{NRS} \neq 1)\end{array}$} & \multirow[t]{2}{*}{$\mathrm{p}$ value } \\
\hline & $\mathrm{n}$ & $\%$ & \\
\hline \multicolumn{4}{|l|}{ Group 1 propofol } \\
\hline monosedation, $\mathrm{n}=20$ & 0 & 0 & NS \\
\hline \multicolumn{4}{|l|}{ Group 2 propofol +} \\
\hline meperidine, $\mathrm{n}=114$ & \multicolumn{3}{|c|}{ Group 3 midazolam +} \\
\hline $\begin{array}{ll}\text { Group } 3 & \begin{array}{l}\text { midazolam }+ \\
\text { meperidine, } \mathrm{n}=147\end{array}\end{array}$ & 35 & 23.8 & 0.0812 \\
\hline \multicolumn{4}{|l|}{ Group 4 other sedation agents/ } \\
\hline no sedation, $\mathrm{n}=12$ & 5 & 41.7 & 0.0191 \\
\hline
\end{tabular}

\section{Patient Satisfaction, Fear and Pain}

Influencing factors of patient satisfaction and memory were as follows: (a) fear and pain during endoscopy reduced patient satisfaction highly significantly $(\mathrm{p}=0.001$ and $\mathrm{p}=0.0001$ ), and (b) 36 (questionnaire after endoscopy) and 39 (interview via telephone) patients complained about the waiting time (12.3 and 15.7\%), however waiting time reduced patient satisfaction non-significantly ( $\mathrm{p}=0.19 ; \mathrm{p}=0.99)$.

Influencing factors or patient's fear and pain yielded the following significances: (a) sedation with the combination midazolam + meperidine increased fear during the endoscopic procedure significantly $(\mathrm{p}=0.082)$ in comparison to monosedation with propofol (table 1); (b) all patients in the sedation group, propofol monoseda-
Table 2. Pain during endoscopy in different sedation groups

\begin{tabular}{|c|c|c|c|}
\hline \multirow[t]{2}{*}{ Sedation group } & \multicolumn{2}{|c|}{$\begin{array}{l}\text { Patients having pain } \\
\text { during endoscopy } \\
(\mathrm{NRS} \neq 1)\end{array}$} & \multirow[t]{2}{*}{$\mathrm{p}$ value } \\
\hline & $\mathrm{n}$ & $\%$ & \\
\hline \multicolumn{4}{|l|}{ Group 1 propofol } \\
\hline monosedation, $\mathrm{n}=18$ & 0 & 0 & NS \\
\hline $\begin{array}{ll}\text { Group } 2 & \text { propofol }+ \\
\text { meperidine, } n=116\end{array}$ & 19 & 164 & $<0.0001$ \\
\hline Group 3 midazolam + & & & \\
\hline meperidine, $\mathrm{n}=146$ & 13 & 8.9 & $<0.0001$ \\
\hline $\begin{array}{cl}\text { Group } 4 & \begin{array}{l}\text { other sedation agents/ } \\
\text { no sedation, } \mathrm{n}=12\end{array}\end{array}$ & 2 & 16.7 & $<0.0001$ \\
\hline
\end{tabular}

tion, indicated significantly ( $\mathrm{p}<0.0001)$ less pain in comparison to the other sedation groups (table 2), and (c) waiting time did not increase fear before or during the procedure.

\section{Cardiovascular Events}

Propofol/meperidine and midazolam/meperidine in combination increased the probability for an incident during and after the procedure in comparison to monosedation with propofol $(\mathrm{p}=0.005$ and $\mathrm{p}=0.039)$.

\section{Long-Term Sedation-Related Adverse Events}

Long-term sedation-related adverse events were defined as side effects, which occurred in timed distance to the procedure over a period of 30 days (mean value). 
These included gastrointestinal disorders, pain, cardiovascular problems and others like allergic reactions, fever, croakiness and swallowing troubles. Propofol and meperidine in combination reduced the probability for an adverse event in comparison to propofol in monosedation $(\mathrm{p}=0.088)$; midazolam and meperidine in combination reduced the probability for pain as a long-term sedationrelated adverse event significantly $(\mathrm{p}=0.019)$. Minor sedation-related adverse events occurred in $31.7 \%$ of cases $(\mathrm{n}=78)$.

\section{Acceptance for Repeat Endoscopies}

Pain and fear during the endoscopic procedure reduced the acceptance for repeat endoscopies significantly $(\mathrm{p}=0.046$ and $\mathrm{p}=0.002$, respectively).

\section{Dosage of Sedation}

No significant differences were found regarding the doses of sedative agents and number of precedent procedures, fear before the last examination, fear and pain before and during the upcoming endoscopy. By drawing a comparison between the groups of propofol in monosedation and propofol in combination with meperidine, we observed significantly lower doses for propofol in monosedation $(\mathrm{p}=0.066)$.

\section{Discussion}

Despite implementation of screening and surveillance programs, only $30 \%$ of the population participates in these programs $[2,3]$. A German feedback report about the participation rates for endoscopies in doctors' practices in 2012 showed that only up to $21 \%$ of eligible patients between 55 and 74 years of age attended the 10 -yearly screening colonoscopy. This report also made a point of the decrease of screening colonoscopy since 2004 from $38 \%$ to about $20 \%$ [16].

Additionally, there is still a worldwide inconsistency of sedation practices in reference to the sedation agents that could be administered. An important influencing factor of patient satisfaction is represented by the choice of sedative agent and combination of sedative agents $[15,17$, 18]. According to a West European study performed in 2010 by Riphaus et al. [15], $41 \%$ of colonoscopies were performed with propofol, $31 \%$ with a combination of benzodiazepines and opioids, $14 \%$ with benzodiazepines alone, and $14 \%$ of the procedures without sedation agents. The extraordinary trend of sedation in screening colonoscopies towards propofol is emphasized by a nation- wide survey 4 years after implementation of the S3 guidelines for sedation in gastroenterology in 2007. In total, the examinations under sedation increased from 74 to $82 \%$ for esophagogastroduodenoscopies and from 87 to $91 \%$ for colonoscopies. Remarkable was the increase of propofol sedation from 74 to $97 \%$, whereas sedation with midazolam declined from 82 to $69 \%$. The most common type of sedation remained the combination of propofol and midazolam (43\%), the sedation with midazolam and meperidine decreased to $3 \%$, propofol in monosedation increased from $11 \%$ in 2007 to $24 \%$ in 2011 [19].

The above-mentioned combination propofol and midazolam was indeed rarely used in our study $(0.3 \%)$. The reasons for this were that every endoscopist has different clinical practice and experience and accordingly chooses the sedative agent, and that the combination of midazolam and propofol is no longer recommended in the current literature. The reasons include an increased risk of cardiovascular complications during and after the endoscopic examination (21.5\% for midazolam/propofol vs. $1.2 \%$ for propofol) [20]. Moreover, a retrospective cohort study by Poulos et al. [17] compared the sedation groups propofol and midazolam/fentanyl/propofol (MFP). MFP showed a longer procedure time (MFP $15 \pm$ $0.004 \mathrm{~min}$ vs. propofol $13 \pm 0.36 \mathrm{~min})$, prolonged recovery time after the procedure (MFP $15 \pm 9$ min vs. propofol $9 \pm 8 \mathrm{~min}$ ) and more discomfort in comparison to monosedation with propofol. In addition to this, Grünhage et al. [21] compared the CFF (critical flitter frequency = analysis to assess the time-dependent effect of sedation on brain function) in patients receiving the sedation regimes propofol (P) or propofol/midazolam (PM). The drop in CFF results was more distinctive in the PM group than the P group ( 30 min CFF: 37.6 vs. $42.2 \mathrm{~Hz}$ ) and driving capability was ensured in the $P$ group after $60 \mathrm{~min}$, whereas sedation with propofol and midazolam had much longer-lasting effects.

As shown in previous studies, important factors for non-participation in endoscopic examinations are fear of the procedure and of side effects as well as pain during and after the endoscopy $[10,11]$. Accordingly, the aim of our study was to identify the type of sedation with the patient's best satisfaction to potentially increase the participation rates for endoscopic screening and surveillance programs and to evaluate factors influencing patient satisfaction for improving quality standards. We found that propofol in monosedation should preferably be used for patient sedation in screening and surveillance endoscopies. Sipe et al. [22] compared the sedation with propofol and midazolam/meperidine. Patient satisfaction was sig-
46

Digestion 2014;90:42-48 DOI: $10.1159 / 000363567$
Kilgert/Rybizki/Grottke/Neurath/ Neumann 
nificantly better for monosedation with propofol. A randomized controlled trial by VanNatta et al. [23] showed that the group with propofol sedation remembered the procedure less often and had less pain than in the combination group.

As shown in our study, significantly less pain and fear of the procedure were reported for monosedation with propofol. Consequently, we observed better patient satisfaction for monosedation in comparison to the combination groups. In addition to this, a study by Ussui et al. [11] in 2013 underlined that reduced abdominal pain is even an important factor for patients to accept repeat procedures. Similar results were observed in our prospective study: fear and pain during previous examinations increased the fear of upcoming endoscopies, reduced patient satisfaction and the acceptance for repeat examinations.

According to Riphaus et al. [8] and several other studies $[18,24,25]$, the utilization of a combination of sedation agents yielded more often respiratory and cardiovascular side effects than monotherapy. As cardiovascular events and side effects decrease patient satisfaction and the acceptance for repeat endoscopies, the rate for cardiovascular events should be held as low as possible [11].

The probability for minor long-term sedation-related adverse events (e.g. gastrointestinal disorders, pain, cardiovascular problems and others like allergic reaction, fever, croakiness and swallow troubles) was higher for pro- pofol in monosedation, which is in contrast to the probability for cardiovascular side effects. In our opinion, the fact that propofol in monosedation reduces patient pain and fear and improves general satisfaction rates is predominant to the probability for the appearance of longterm minor sedation-related side effects which were even rarer. In contrast to other studies, propofol in monosedation resulted in a decrease of propofol usage in comparison to the other sedation groups $[8,18,26]$. Consequently, there is even no reason to prefer combination therapy to monotherapy with propofol regarding saving propofol for sedation.

In conclusion, propofol in monosedation should preferably be used for patient sedation in screening or surveillance programs, as it reduces fear and pain during endoscopies in comparison to other sedation types. This fact yields in better patient satisfaction for endoscopic procedures. In our prospective study we have shown that propofol, when administered in monosedation, yielded the highest patient satisfaction rates and may therefore have the potential to improve patient acceptance rates for endoscopic procedures.

\section{Disclosure Statement}

The authors have no conflicts of interests to disclose.

\section{References}

$>1$ Boyle P, Langman JS: ABC of colorectal cancer: Epidemiology. BMJ 2000;321:805808.

$>2$ Rex DK, Johnson DA, Anderson JC, Schoenfeld PS, Burke CA, Inadomi JM, et al: American College of Gastroenterology guidelines for colorectal cancer screening 2009 (corrected). Am J Gastroenterol 2009; 104:739-750.

-3 Brenner H, Hoffmeister M, Brenner G, Altenhofen L, Haug U: Expected reduction of colorectal cancer incidence within 8 years after introduction of the German screening colonoscopy programme: estimates based on $1,875,708$ screening colonoscopies. Eur J Cancer 2009;45:2027-2033.

$\checkmark 4$ Baudet JS, Diaz-Bethencourt D, Aviles J, Aguirre-Jaime A: Minor adverse events of colonoscopy on ambulatory patients: the impact of moderate sedation. Eur J Gastroenterol Hepatol 2009;21:656-661.

$>5$ Abraham NS, Fallone CA, Mayrand S, Huang J, Wieczorek P, Barkun AN: Sedation versus no sedation in the performance of diagnostic upper gastrointestinal endoscopy: a Canadian randomized controlled cost-outcome study. Am J Gastroenterol 2004;99:1692-1699.

6 Lee MG, Hanna W, Harding H: Sedation for upper gastrointestinal endoscopy: a comparative study of midazolam and diazepam. Gastrointest Endosc 1989;35:82-84.

7 Cohen LB, Delegge MH, Aisenberg J, Brill JV, Inadomi JM, Kochman ML, et al: AGA Institute review of endoscopic sedation. Gastroenterology 2007;133:675-701.

$>8$ Riphaus A, Wehrmann T, Weber B, Arnold J, Beilenhoff U, Bitter $\mathrm{H}$, et al: $\mathrm{S} 3$ guidelines - sedation in gastrointestinal endoscopy (in German). Z Gastroenterol 2008;46:1298-1330.

-9 De Wijkerslooth TR, de Haan MC, Stoop EM, Bossuyt PM, Thomeer M, van Leerdam ME, et al: Reasons for participation and nonparticipation in colorectal cancer screening: a randomized trial of colonoscopy and CT colonography. Am J Gastroenterol 2012;107: 1777-1783.

10 Baudet JS, Aguirre-Jaime A: The sedation increases the acceptance of repeat colonoscopies. Eur J Gastroenterol Hepatol 2012;24: 775-780.
11 Ussui VM, da Silva AL, Borges LV, da Silva JG, Zeitune JM, Hashimoto CL: What are the most important factors regarding acceptance to the colonoscopy? Study of related tolerance parameters. Arq Gastroenterol 2013;50:2330.

12 Froehlich F, Harris JK, Wietlisbach V, Burnand B, Vader JP, Gonvers JJ, et al: Current sedation and monitoring practice for colonoscopy: an International Observational Study (EPAGE). Endoscopy 2006;38:461469.

13 Porostocky P, Chiba N, Colacino P, Sadowski $\mathrm{D}$, Singh $\mathrm{H}$ : A survey of sedation practices for colonoscopy in Canada. Can J Gastroenterol 2011;25:255-260.

14 Ladas SD, Satake Y, Mostafa I, Morse J: Sedation practices for gastrointestinal endoscopy in Europe, North America, Asia, Africa and Australia. Digestion 2010;82:74-76.

$>15$ Riphaus A, Macias-Gomez C, Deviere J, Dumonceau JM: Propofol, the preferred sedation for screening colonoscopy, is underused. Results of an international survey. Dig Liver Dis 2012;44:389-392.
Assessment of Sedation-Related Adverse

Events and Patient Satisfaction 
16 Versorgung Zfdk. Feedback-Bericht Früherkennungs-Koloskopie. Erstellt im Auftrag der Kassenärztlichen Bundesvereinigung und des GKV-Spitzenverbandes; in Praxis Dr. med. Helmut Neumann LS, 32105 Bad Salzuflen, editor, 2012.

17 Poulos JE, Kalogerinis PT, Caudle JN: Propofol compared with combination propofol or midazolam/fentanyl for endoscopy in a community setting. AANA J 2013;81:31-36.

18 Moerman AT, Struys MM, Vereecke HE, Herregods LL, De Vos MM, Mortier EP: Remifentanil used to supplement propofol does not improve quality of sedation during spontaneous respiration. J Clin Anesth 2004; $16: 237-243$.
19 Riphaus A, Geist F, Wehrmann T: Endoscopic sedation and monitoring practice in Germany: re-evaluation from the first nationwide survey 3 years after the implementation of an evidence and consent based national guideline. Z Gastroenterol 2013;51:10821088.

20 Pascual MG, Zayas Berbes M, Saez Banos M, Abreu Vazquez Mdel R, Martinez Leyva L: Propofol versus midazolam and pethidine in the colonoscopy realization (in Spanish). Acta Gastroenterol Latinoam 2011;41:214-220.

21 Grünhage F, Seegmüller A, Lammert F: Effect of different sedation on critical flicker frequency, a diagnostic tool for minimal encephalopathy. Z Gastroenterol 2013;51:K261.

22 Sipe BW, Rex DK, Latinovich D, Overley C, Kinser K, Bratcher L, et al: Propofol versus midazolam/meperidine for outpatient colonoscopy: administration by nurses supervised by endoscopists. Gastrointest Endosc 2002; 55:815-825.
23 VanNatta ME, Rex DK: Propofol alone titrated to deep sedation versus propofol in combination with opioids and/or benzodiazepines and titrated to moderate sedation for colonoscopy. Am J Gastroenterol 2006;101:2209-2217.

24 DiPalma JA, Herrera JL, Weis FR, DarkMezick DL, Brown RS: Alfentanil for conscious sedation during colonoscopy. South Med J 1995;88:630-634.

25 Rembacken BJ, Axon AT: The role of pethidine in sedation for colonoscopy. Endoscopy 1995;27:244-247.

26 Cordruwisch W, Doroschko M, Wurbs D: Deep sedation in gastrointestinal endoscopic interventions: safety and reliability of a combination of midazolam and propofol (in German). Dtsch Med Wochenschr 2000;125:619-622. 